\title{
Uma abordagem para integração do Moodle com o framework Shiny para Learning Analytics
}

\author{
João C. Sedraz Silva ${ }^{1}$, Jorge L. C. Ramos ${ }^{1}$, Rodrigo Lins Rodrigues ${ }^{2}$, Fernando d. F. \\ de Souza $^{3}$, Alex Sandro Gomes 3 , Hugo L. V. Souza ${ }^{3}$ \\ ${ }^{1}$ Universidade Federal do Vale do São Francisco \\ Juazeiro - BA - Brasil \\ ${ }^{2}$ Universidade Federal Rural de Pernambuco \\ Recife-PE - Brasil \\ ${ }^{3}$ Universidade Federal de Pernambuco \\ Recife-PE - Brasil \\ rodrigo.linsrodrigues@ufrpe.br, \{joao.sedraz, jorge.cavalcanti\}@univasf. \\ edu.br, \{asg,fdfd\}@cin.ufpe.br, hvlsouza@gmail.com
}

\begin{abstract}
This study aimed to present a Moodle LMS integration approach with the framework Shiny for the development of Learning Analytics. In order to demonstrate the feasibility of this approach, a web application able to collect and process data from the LMS was implemented. The results show that the integration of Moodle with Shiny expands the possibilities of Learning Analytics, with the freedom to produce solutions that can be tailored to specific educational contexts.
\end{abstract}

Resumo. O presente trabalho teve por objetivo apresentar uma abordagem da integração do LMS Moodle com o framework Shiny para o desenvolvimento de Learning Analytics. Como forma de evidenciar a viabilidade dessa abordagem, foi implementada uma aplicação web capaz de coletar e tratar os dados do LMS. Os resultados obtidos demonstram que a integração do Moodle com o Shiny amplia as possibilidades de Learning Analytics, com a liberdade de produzir soluções que podem ser adaptadas para contextos educacionais especificos.

\section{Introdução}

Com a ampliação e facilidade do acesso à internet, tornou-se possível a utilização de diversos sistemas web. Dentre esses, no ramo educacional, destacam-se os Sistemas de Gestão da Aprendizagem (LMS, do inglês Learning Management Systems).

O uso de LMS viabiliza a interação em cursos a distância e cria novas possibilidades para os cursos presenciais, permitindo aos professores e estudantes oportunidades para desenvolverem novos métodos de ensino e aprendizagem [Silva et al. 2015].

Os LMS registram as interações dos estudantes no ambiente virtual, favorecendo a obtenção de medidas quantitativas, que podem ser analisadas para proporcionar uma melhor compreensão sobre a efetividade das estratégias pedagógicas. Também conhecida por Learning Analytics (LA), esse tipo de análise vem se definindo como uma das áreas das tecnologias educacionais com maior crescimento [Nunes 2015]. 
O uso de LA tem crescido por quatro motivos principais: o aumento substancial na quantidade de dados, a melhoria no formato dos dados, os avanços na computação e a maior disponibilidade de ferramentas para as análises [Siemens e Baker 2012].

Apesar da atenção dada à LA como um conceito e o desenvolvimento de diversos métodos de análises, geralmente, os educadores enfrentam dificuldades para encontrar ferramentas abertas e integradas com os LMS, o que é um obstáculo para a realização de avaliações variadas e complexas. No contexto do Brasil, soma-se a essas dificuldades, o número reduzido de trabalhos dedicados a essa área [Nunes 2015]

Assim, como uma contribuição aos estudos sobre LA, o objetivo deste trabalho é apresentar uma abordagem da integração do LMS Moodle com o framework Shiny para o desenvolvimento de Learning Analytics.

A escolha do Moodle deve-se a presença mundial da plataforma, com 76.781 instâncias registradas em 231 países [Moodle 2016], e também a sua liderança no cenário nacional, representando o LMS mais adotado pelas instituições públicas brasileiras [Gluz et al. 2011; Silva et al. 2015].

Além desta introdução, o trabalho está organizado com mais cinco seções, que apresentam a fundamentação deste artigo, o método utilizado, a discussão dos resultados e as considerações finais da pesquisa.

\section{Learning Analytics}

A Learning Analytics (LA) é defina pela Society for Learning Analytics Research (SoLAR) como a medição, coleta, análise e comunicação de dados sobre os estudantes e seus contextos, para compreender e otimizar a aprendizagem e o ambiente em que ela ocorre. Dentro dessa definição, destaca-se que o maior propósito da LA é orientar a tomada de decisão sobre as estratégias para promover intervenções adequadas junto aos estudantes em situação de risco [Siemens e Baker 2012].

A partir da análise dos rastros digitais e analógicos que os estudantes e professores deixam durante o processo de ensino e aprendizagem, os pesquisadores de LA buscam extrair padrões. Esses padrões podem ser obtidos por técnicas de mineração de dados, para auxiliar na recomendação de pessoas, recursos ou atividades que favoreçam o aprendizado [Nunes 2015].

Mas, cabe ressaltar que LA não está centrada em delegar o controle a uma espécie de sistemas de tutores inteligentes, mas em conceder o controle aos usuários, fornecendo consciência e suporte para a melhoria do processo educativo. Em razão disso, a visualização de dados educacionais é considerada como uma alternativa muito importante para a LA [Duval 2011].

\subsection{Visualização de dados educacionais}

Os dados dos ambientes virtuais de aprendizagem ainda são pouco explorados para gerar indicadores relevantes dos cursos. Quando devidamente tratados e analisados, permitem novos conhecimentos acerca das turmas, do engajamento e da motivação dos estudantes, fatores que influenciam a aprendizagem online [Cavalcanti et al. 2014]. 
V Congresso Brasileiro de Informática na Educação (CBIE 2016)

Anais dos Workshops do V Congresso Brasileiro de Informática na Educação (CBIE 2016)

As visualizações de dados educacionais permitem que o professor tenha uma visão geral do comportamento dos estudantes que, essencialmente, busca remediar a "condução cega" muitas vezes enfrentada na atividade docente [Duval 2011].

Nesse sentido, técnicas de visualização de dados podem apoiar a análise por especialistas humanos, exibindo sínteses em forma de gráficos ou imagens. Essas imagens possibilitam uma rápida interpretação e percepção de indicativos importantes dos cursos e seus discentes [Ramos et al. 2016].

\subsection{Soluções para a visualização de dados educacionais}

A literatura aponta algumas soluções para a visualização de dados educacionais. A Figura 1 ilustra três dessas soluções.

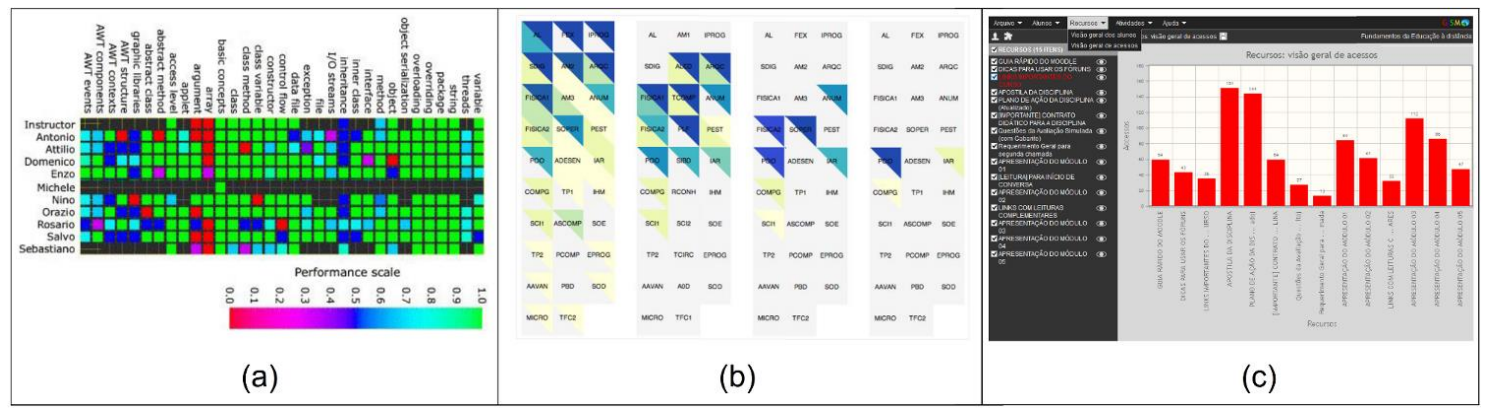

Figura 1. Imagens de soluções utilizadas para a visualização de dados educacionais: (a) Matriz Cognitiva do CourseVis, (b) Representação multimatricial do EduVis, (c) Relatório de acesso aos recursos do Gismo.

O CourseVis permite a visualização dos dados de cursos online dentro do ambiente virtual WebCT. Essa ferramenta é usada como uma extensão do próprio LMS e permite explorar os dados de maneira interativa, além de possibilitar a manipulação dos mesmos a partir de diferentes mecanismos de visualização. Destaca-se entre esses mecanismos, a Matriz Cognitiva (Figura 1a), com a representação do desempenho dos estudantes em cada atividade de aprendizagem [Mazza e Dimitrova 2007].

A solução EduVis proporciona uma visualização coordenada das disciplinas por meio de mapas de cores (Figura 1b), associados a padrões específicos observados nas componentes curriculares [Gama et al. 2014].

O GISMO' (Graphical Interactive Student Monitoring Tool for Moodle) é uma aplicação open source que funciona como uma extensão do Moodle, podendo ser adicionada ao ambiente para gerar gráficos das interações dos estudantes (Figura 1c) com os recursos e as atividades disponíveis no LMS [Ramos et al. 2016].

Essas soluções cumprem seu papel de transformar um grande volume de dados em algo mais compreensível. Porém, são limitadas em termos de customização e não oferecem recursos adequados para várias situações. A abordagem descrita neste estudo amplia as possibilidades de LA por meio de uma solução aberta, que permite o desenvolvimento de recursos para o atendimento de requisitos identificados em contextos educacionais específicos.

\footnotetext{
${ }^{1} \mathrm{http}: / /$ gismo.sourceforge.net/
} 
V Congresso Brasileiro de Informática na Educação (CBIE 2016)

Anais dos Workshops do V Congresso Brasileiro de Informática na Educação (CBIE 2016)

\section{Framework Shiny}

O Shiny é um pacote do software estatístico R criado para facilitar o desenvolvimento de aplicações web. Com ele, é possível construir interfaces interativas de maneira simples, sem a necessidade de conhecimentos avançados em tecnologias de desenvolvimento web, como HTML, CSS ou JavaScript. Isso é alcançado por meio de componentes pré-construídos, que facilitam a implementação de interfaces com o mínimo de esforço [Bavaresco e Rosa 2015].

As aplicações desenvolvidas a partir do framework Shiny são multiplataforma e podem ser executadas localmente, pelo ambiente RStudio, ou por um navegador web, quando hospedadas em um servidor remoto.

O Shiny combina o poder computacional do software R com a interatividade da web moderna, tornando-se uma opção interessante para uso em computação científica. $\mathrm{O}$ site oficial do framework ${ }^{2}$ disponibiliza diversos tutoriais, artigos e exemplos de aplicações, que podem ser utilizados como guias para o desenvolvimento de novas soluções de LA, como a apresentada nas próximas seções deste trabalho.

\section{Método}

Neste artigo, foi conduzido um estudo de caso que, segundo Yin (2013), justifica-se em situações contemporâneas em que é necessária a investigação profunda de uma unidade de análise. O trabalho descreve uma abordagem para o suporte de Learning Analytics, realizada a partir da integração do Moodle e o framework Shiny.

Como forma de materializar a integração do LMS com o framework, foi desenvolvida uma aplicação web. Para demonstrar as funcionalidades implementadas na aplicação, foram utilizados dados registrados na instância de testes do Moodle, disponível na página oficial de distribuição do sistema ${ }^{3}$.

O objetivo foi apresentar uma abordagem da integração do LMS Moodle com o framework Shiny para o desenvolvimento de Learning Analytics.

Para o atendimento deste objetivo, foi implementada uma solução a partir do framework Shiny, que realiza coleta e processamento de dados registrados no Moodle. Durante o desenvolvimento, além da instalação dos pacotes requeridos (Figura 4), foram especificados três arquivos adotados pelo framework: global.R, ui.R e server.R.

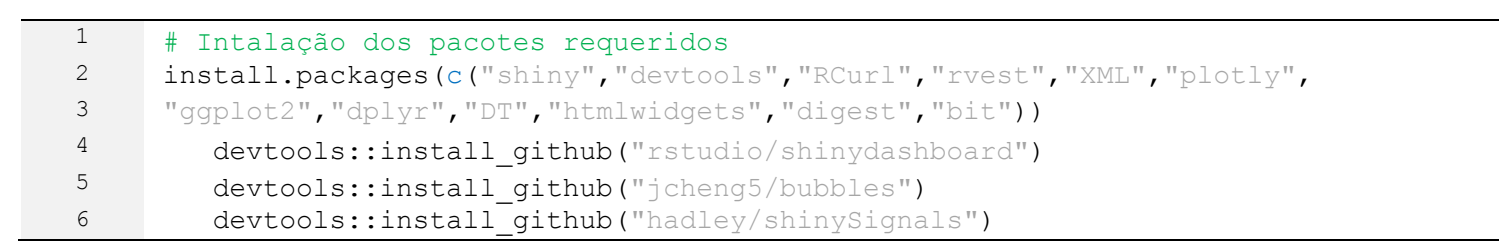

Figura 2. Script em R para a instalação dos pacotes requeridos na aplicação.

A Figura 3, apresenta o código fonte do arquivo global.R. Ele agrega os recursos que são utilizados pelos outros componentes da aplicação (ui.R e server.R). As primeiras linhas são reservadas para as configurações básicas (endereço eletrônico do

\footnotetext{
2 http://shiny.rstudio.com/

${ }^{3} \mathrm{http}: / /$ school.demo.moodle.net
} 
V Congresso Brasileiro de Informática na Educação (CBIE 2016)

Anais dos Workshops do V Congresso Brasileiro de Informática na Educação (CBIE 2016)

LMS, código de identificação da disciplina, nome do usuário e senha). Entre as linhas 7 e 10, são carregados os pacotes requeridos. Nas linhas 12 e 13, são indicadas as partes que compõem o caminho de acesso aos registros da instância do Moodle. Na sequência, são listados comandos para conexão, coleta de dados e ajuste no formato das datas.

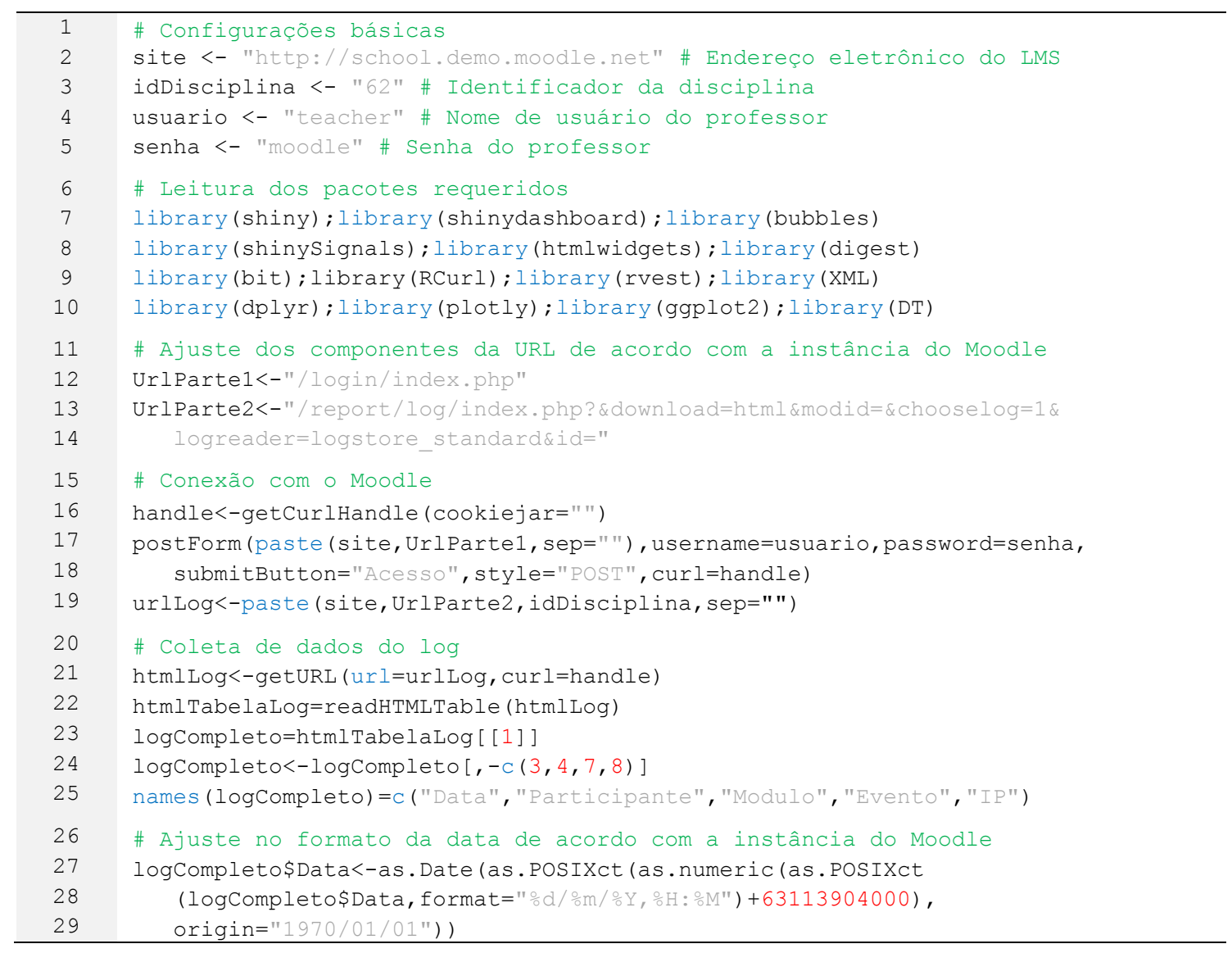

Figura 3. Script em $\mathbf{R}$ do arquivo global. $R$.

O arquivo ui.R é o responsável pela geração da interface gráfica (Figura 4). Nesse arquivo foi adotado o modelo Shiny Dashboard ${ }^{4}$. A interface foi projetada em duas colunas. Na coluna da esquerda, foram incorporados o menu principal e os campos para a seleção do período de busca. Já na coluna da direita, foi reservado o espaço para a exibição dos dados processados. Os detalhes da interface são apresentados na seção de resultados deste trabalho.

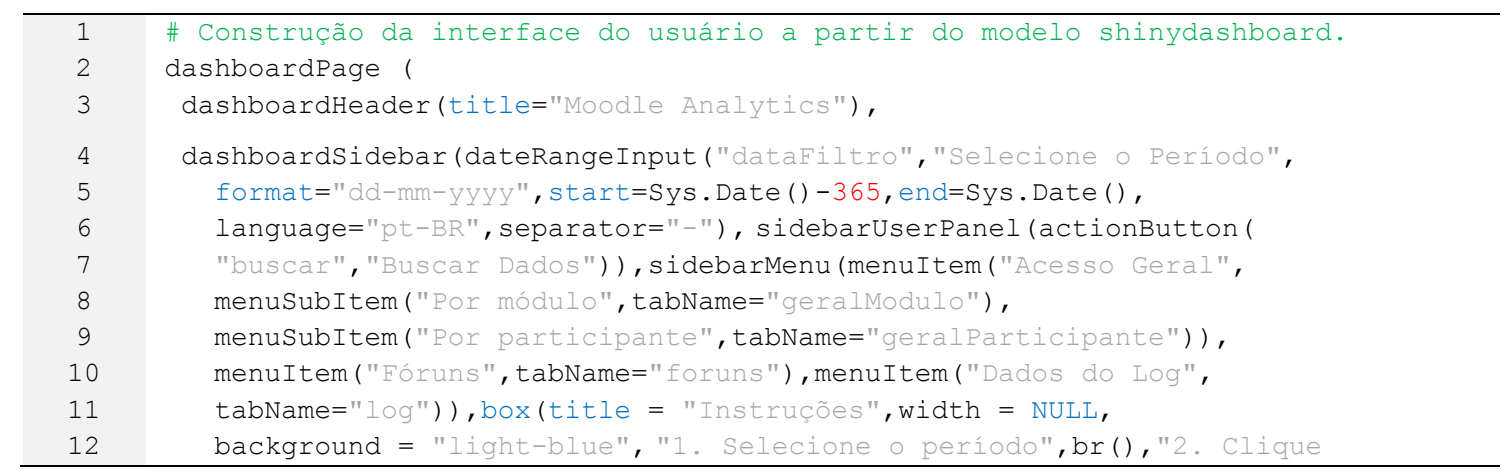

\footnotetext{
${ }^{4} \mathrm{O}$ modelo Shiny Dashboard pode ser acessado em https://rstudio.github.io/shinydashboard/structure.html.
} 
V Congresso Brasileiro de Informática na Educação (CBIE 2016)

Anais dos Workshops do V Congresso Brasileiro de Informática na Educação (CBIE 2016)

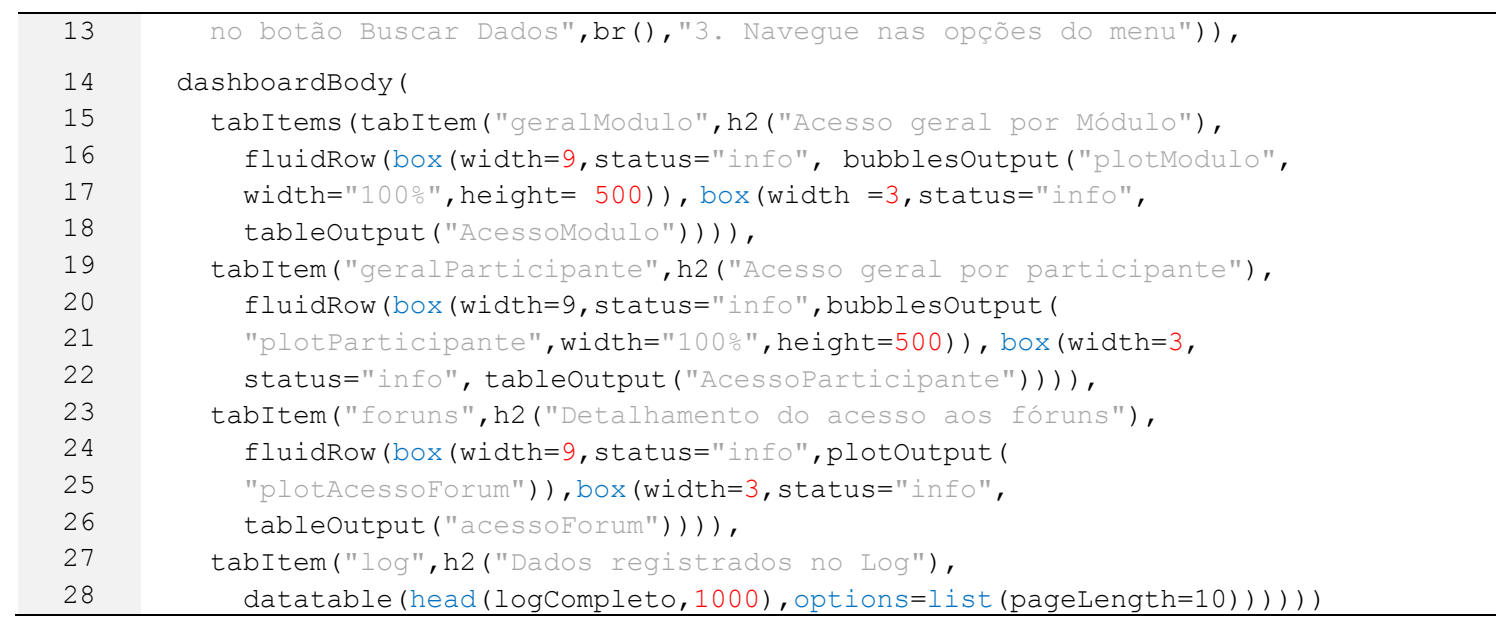

Figura 4. Script em $\mathrm{R}$ do arquivo ui.R.

A Figura 5, detalha o arquivo server.R, que é o responsável pelo processamento dos dados. Nas linhas 4 e 5 , o código apresenta as variáveis que recebem os valores das datas que delimitam o período de observação dos dados selecionado pelo usuário. De acordo com o período definido, os dados são filtrados pelos comandos apresentados entre as linhas 7 e 10. Após esse filtro inicial, os dados são tratados com o propósito de subsidiar três análises.

Da linha 12 a 18, os dados são sintetizados em um gráfico e em uma tabela com informações que permitem a análise dos registros de acesso por estudantes. $\mathrm{O}$ código entre as linhas 20 e 24 executa passos semelhantes, mas realiza o processamento com foco na análise dos registros de acesso por módulo.

Por fim, da linha 26 a 36, são processados os dados dos fóruns de discussão. $\mathrm{O}$ resultado desse processamento é um gráfico e uma tabela, que podem ser utilizados para a análise da participação de cada usuário nos fóruns, a partir do número de visualizações e de mensagens criadas.

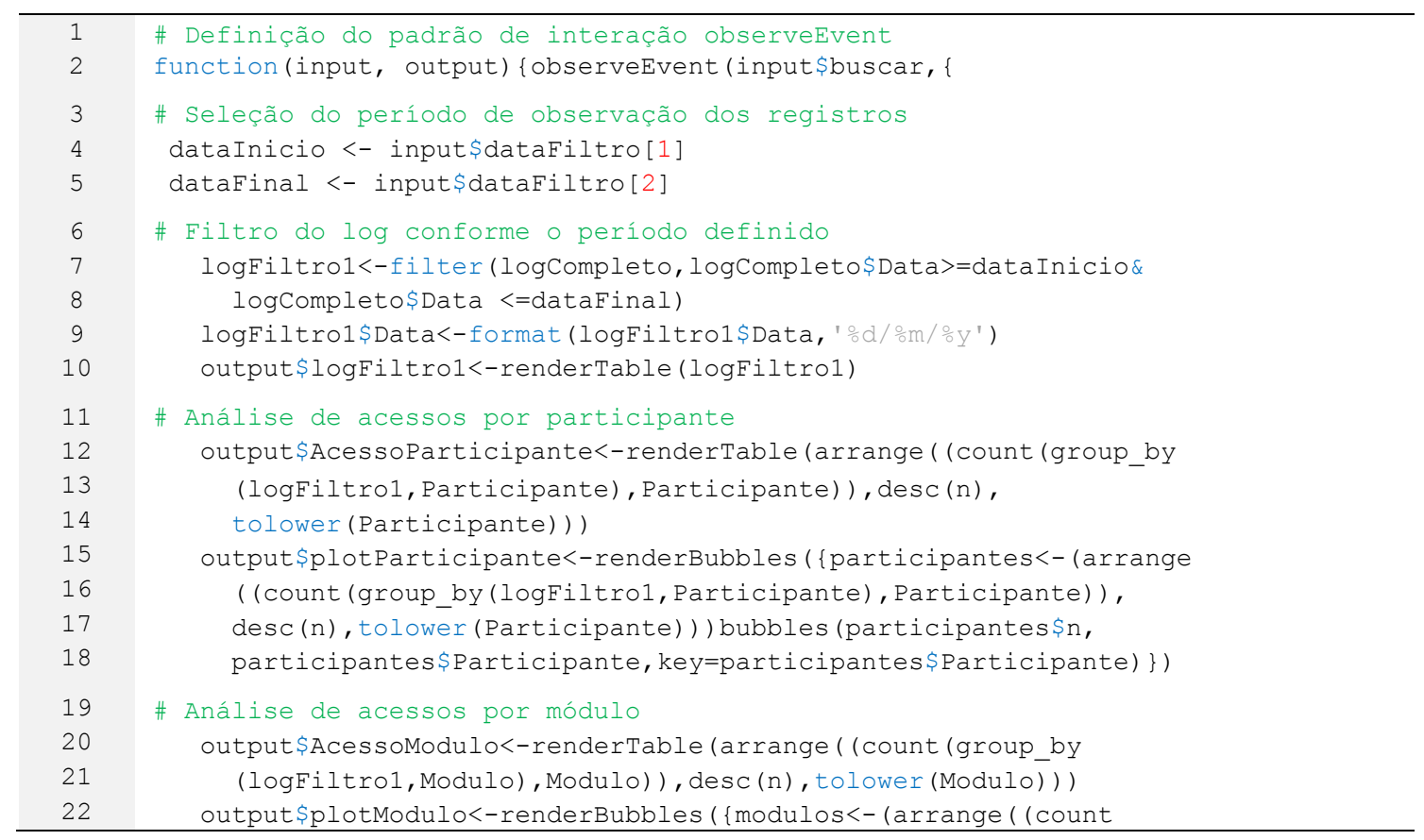


V Congresso Brasileiro de Informática na Educação (CBIE 2016)

Anais dos Workshops do V Congresso Brasileiro de Informática na Educação (CBIE 2016)

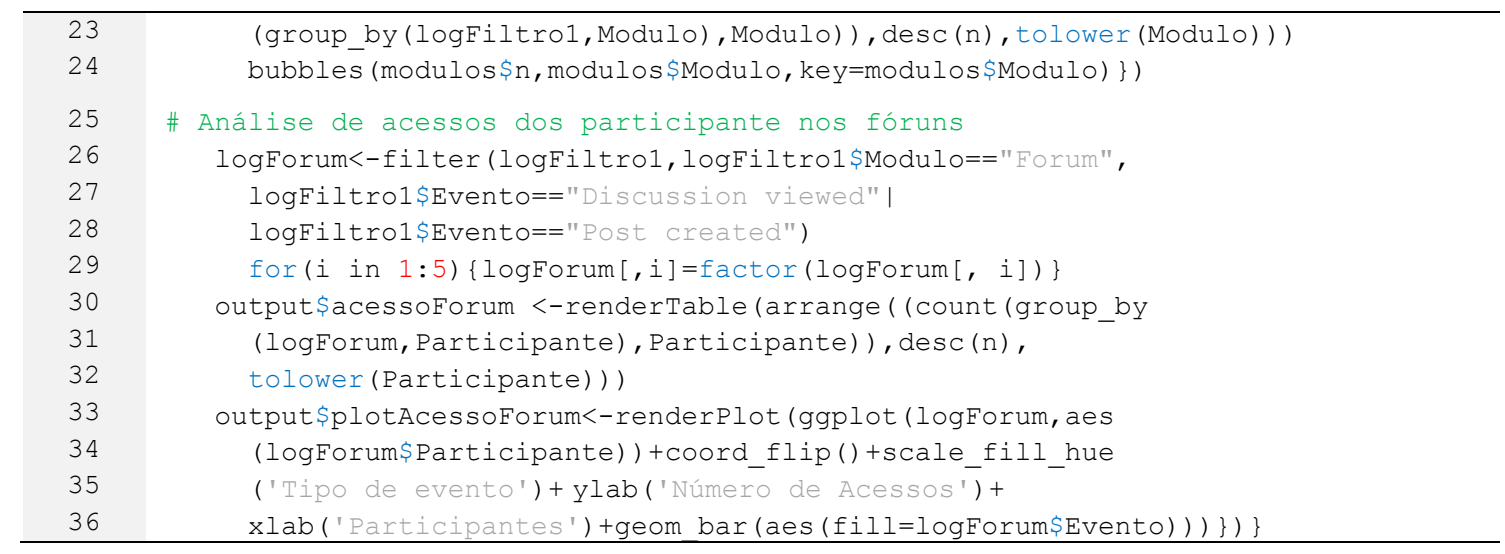

Figura 5. Script em $\mathbf{R}$ do arquivo server. $R$.

Os arquivos ui.R e server.R podem ser incrementados com novas funcionalidades para permitir outras análises. Mas, em razão da limitação de espaço para a publicação, neste artigo, a aplicação desenvolvida foi dedicada apenas ao apoio das três análises citadas anteriormente. Os resultados obtidos com a implementação da aplicação são destacados na seção a seguir.

\section{Resultados}

$\mathrm{Na}$ aplicação implementada, priorizou-se utilizar elementos de interface simples e diretos, para torná-la mais intuitiva para o usuário. Um pequeno bloco de instruções foi colocado abaixo das opções do menu, destacando o roteiro de utilização da aplicação. A ação básica é escolher o período e solicitar a busca dos dados. A partir disso, todas as visualizações ficam disponibilizadas para as análises.

Para a consulta aos acessos em geral, dois filtros são disponibilizados: por módulo e por participante. A Figura 8 ilustra o resultado na análise geral de todos os acessos aos módulos do curso. Além do gráfico em bolhas, é exibida uma tabela auxiliar com os dados da consulta.

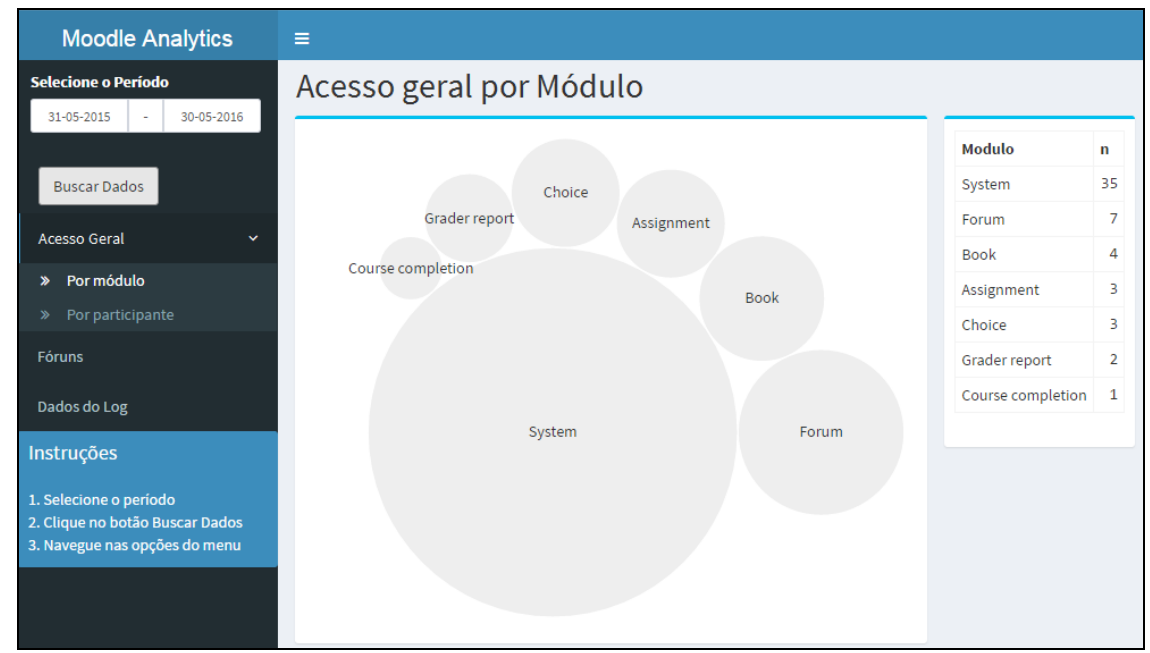

Figura 8. Tela da aplicação com informações dos registros de acesso por módulo.

Esse tipo de visualização permite uma percepção imediata do nível de acessos que recursos ou atividades disponibilizadas no curso estão tendo, possibilitando, por 
V Congresso Brasileiro de Informática na Educação (CBIE 2016)

Anais dos Workshops do V Congresso Brasileiro de Informática na Educação (CBIE 2016)

exemplo, que o professor estimule uma atividade que não atingiu índices satisfatórios de participação.

Ao optar pelo filtro de acessos por participante (Figura 9), são exibidos gráfico e tabela semelhantes, dando ao usuário a rápida informação do nível de participação dos alunos. Com essa percepção da participação por aluno, ações podem ser tomadas, no sentido de motivar alunos com baixa interação, assim como bonificar aqueles que atingem elevados índices de participação no curso.

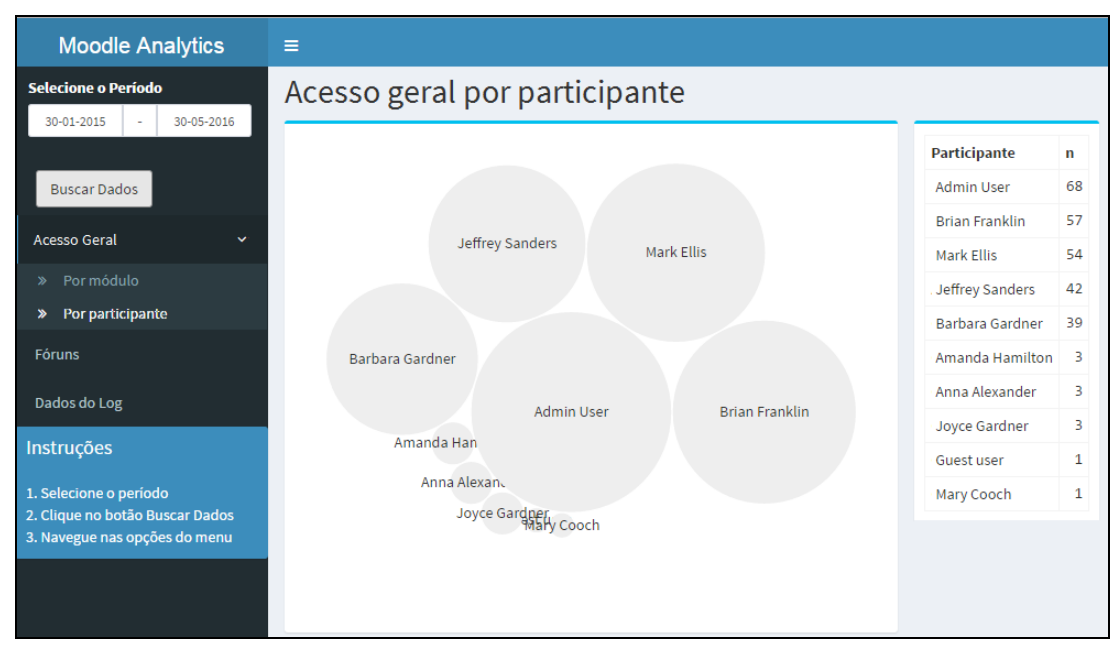

Figura 9. Tela da aplicação com informações dos registros de acesso por participante.

A opção seguinte permite a visualização dos dados agrupados dos fóruns do curso. A Figura 10 exibe o número de acesso por participante, com a distinção entre leitura e criação de postagens. Como os gráficos anteriores, essa visualização permite uma rápida análise da participação dos estudantes nos fóruns, possibilitando ações e decisões do professor em razão desse nível de acesso.

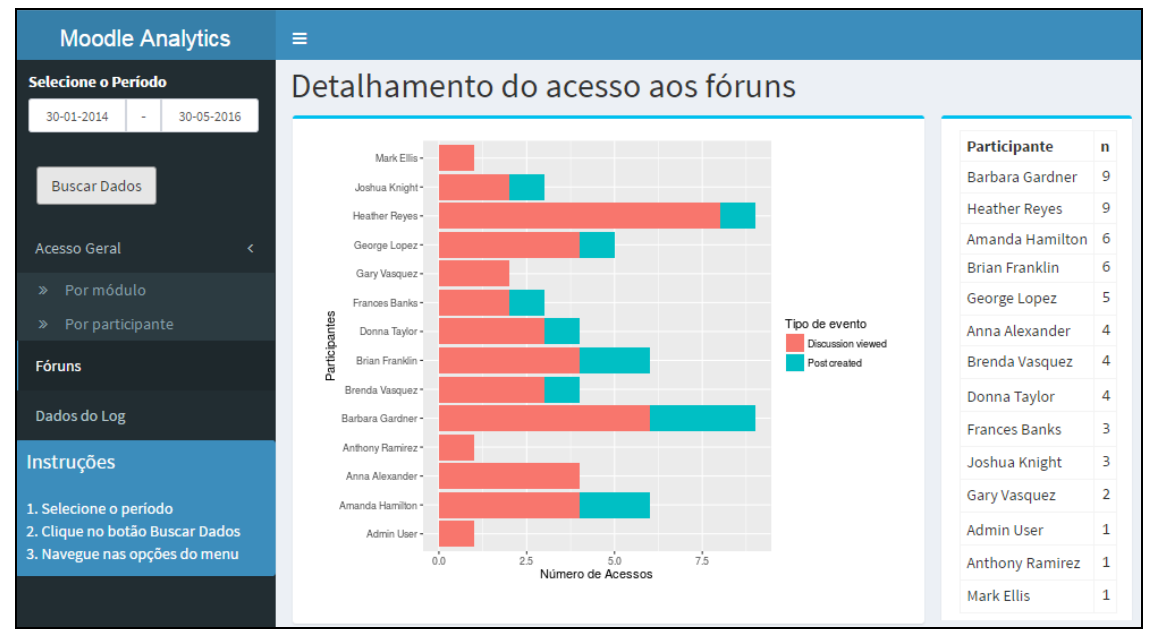

Figura 10. Tela da aplicação com informações sobre os registros de acesso aos fóruns.

Finalmente, a opção Dados do Log (Figura 11) exibe uma listagem de todos os acessos ao curso, com vários parâmetros, permitindo uma busca ou ordenamento dos dados em cada atributo (coluna) da lista. Esse relatório é bastante útil, pois sintetiza informações da extensa tabela " $m d l \_l o g$ " do banco de dados do Moodle, possibilitando consultas otimizadas e rápidas aos acessos do curso. 
V Congresso Brasileiro de Informática na Educação (CBIE 2016)

Anais dos Workshops do V Congresso Brasileiro de Informática na Educação (CBIE 2016)

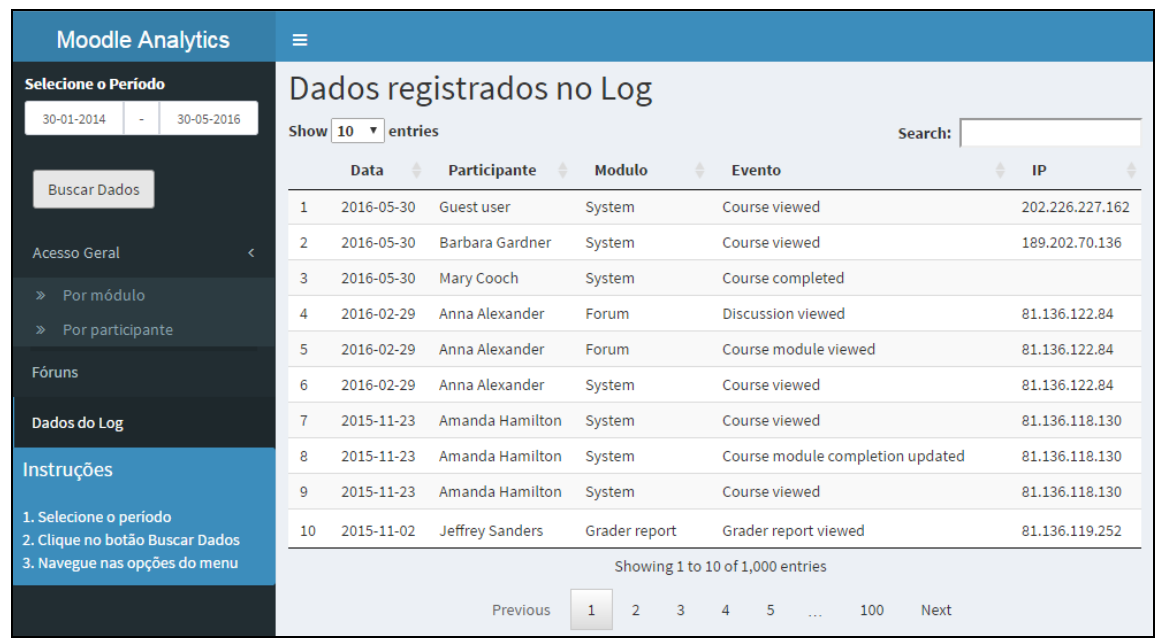

Figura 11. Tela da aplicação que exibe os dados registrados no log do Moodle.

Mesmo tratando de um protótipo inicial, a aplicação pode ser útil para os usuários do Moodle e ainda apresenta um considerável potencial de incremento, pois utiliza o software $\mathrm{R}$ como plataforma de desenvolvimento, o pacote Shiny com constantes evoluções e a própria abrangência do banco de dados do Moodle, com mais de 250 tabelas e relacionamentos diversos.

\section{Considerações finais e trabalhos futuros}

Este trabalho teve por objetivo apresentar uma abordagem da integração do LMS Moodle com o framework Shiny para o desenvolvimento de Learning Analytics. A partir da implementação de uma aplicação web, foi demonstrada a viabilidade de utilização do Shiny na coleta e o tratamento de dados do Moodle, permitindo a produção de visualizações úteis para a análise dos registros das atividades realizadas pelos estudantes no ambiente virtual.

Atualmente, o repositório CRAN (Comprehensive $R$ Archive Network) disponibiliza $8492^{5}$ pacotes desenvolvidos na linguagem $\mathrm{R}$, dedicados a diversos procedimentos de tratamento e exibição de dados. Considerando que as funcionalidades desses pacotes podem ser agregadas com relativa facilidade nas aplicações desenvolvidas com o Shiny, são inúmeras as possibilidades de soluções que podem ser produzidas para a análise de dados do Moodle.

Nas próximas versões da aplicação serão incorporadas novas funcionalidades de pacotes gráficos do $\mathrm{R}$ como o ggplot2, possibilitando uma diversidade bem maior de opções para as visualizações. Também serão disponibilizadas na ferramenta opções para tratamento estatístico dos dados e Educational Data Mining (EDM).

Um dos grandes problemas em torno de Learning Analytics é a falta de clareza sobre o que, exatamente, deve ser medido para obter uma compreensão mais profunda de como a aprendizagem está ocorrendo [Duval 2011]. Cabe ressaltar que este artigo não focou nesse problema, mas, apresentou uma abordagem viável que favorece outras pesquisas nessa direção. Assim, como proposta de trabalhos futuros, pretende-se desenvolver aplicações mais elaboradas e testes de usabilidade em cenários

\footnotetext{
${ }^{5}$ Disponível em https://cran.r-project.org/. Acessado em 31/03/16.
} 
V Congresso Brasileiro de Informática na Educação (CBIE 2016)

Anais dos Workshops do V Congresso Brasileiro de Informática na Educação (CBIE 2016)

educacionais reais, com o propósito de avaliar a efetividade das soluções que integram o Moodle ao framework Shiny para Learning Analytics.

\section{Agradecimentos}

Esta pesquisa é apoiada pela FACEPE-APQ No. 0525-1.03/14. Os autores também agradecem ao apoio da PRPPG/UFRPE (006999/2015-28) e PRPPGI/UNIVASF (Edital 18/2013). Alex Sandro Gomes é bolsista DT Nível 2/CNPq, processos no 310466/20121 e no $475634 / 2013-6$.

\section{Referências}

Bavaresco, J., Rosa, R. (2015). Redes Neurais com Neuralnet e Shiny. In: II Simpósio de Informática IFSUL Passo Fundo - BIG DATA Da infraestrutura à descoberta inteligente de conhecimento. Passo Fundo. p. 55-64.

Cavalcanti, A., dos Santos, N., Ramos, J. L. C, Gomes, A. (2014). Mineração e Visualização de Dados Educacionais: Identificação de Fatores que Afetam a Motivação de Alunos na Educação a Distância. In: Anais do WEIBASE 2014. Feira de Santana-BA.

Duval, E. (2011). Attention please! learning analytics for visualization and recommendation. In: Proceedings of the 1st International Conference on Learning Analytics and Knowledge. ACM. p. 9-17.

Gama, S., Jordão, V., Gonçalves, D. (2014). EduVis: Visualizing Educational Information. NordiCHI '14 Proceedings of the 8th Nordic Conference on HumanComputer Interaction. p. 1011-1014.

Gluz, J. C., Galafassi, C., Penteado, F. (2011). Suporte Técnico/Pedagógico aos OAs pelos AVAs: uma Análise Comparativa das Pesquisas Recentes. SBIE. Aracaju-SE.

Mazza, R., Dimitrova, V. (2007). CourseVis: A graphical student monitoring tool for supporting instructors in web-based distance courses. International Journal of Human-Computer Studies, 65(2), 125-139.

Moodle. Registered Moodle. Disponível em: http://moodle.org/sites. Acesso em: 06/06/16.

Nunes, J. Estado da Arte sobre Analítica da Aprendizagem na América Latina. In: Anais dos Workshops do Congresso Brasileiro de Informática na Educação. 2015. p. 1024.

Ramos, J. L. C, Cavalcanti, A. G. G., Silva, J. C. S., Rodrigues, R. L., Gomes, A. S. (2016). Apresentação e avaliação de ferramenta de visualização gráfica das interações dos estudantes no ambiente Moodle. In Anais do ERBASE. Maceió.

Siemens, G., Baker, R. (2012). Learning analytics and educational data mining: towards communication and collaboration. In: Proceedings of the 2nd international conference on learning analytics and knowledge. ACM. p. 252-254.

Silva, R., Ramos, J. L. C, Silva, J. C. S., Gomes, A. S. (2015). Estudo comparativo entre ambientes virtuais para uso em Blended Learning em uma Universidade. In Anais do Workshop de Informática na Escola (WIE 2015)

Yin, R. K. (2013). “Case study research: Design and methods”. Sage publications. 